\title{
Dimensões da sustentabilidade em bacias hidrográficas: Uma aplicação do 'gráfico de radar' à bacia do Rio Verruga/BA
}

A bacia hidrográfica representa um local de relações intrínsecas do meio antrópico e o meio natural. Por isso, a análise e avaliação de indicadores ambientais são essenciais para a formulação de propostas de políticas sustentáveis para o correto manejo das bacias. Este trabalho teve a intenção de calcular e analisar o índice de sustentabilidade da bacia hidrográfica do Rio Verruga. Para obtenção dos dados e avaliação deste índice relativo à temática da sustentabilidade, Calório (1997) desenvolve uma metodologia de cálculo em que o valor dos índices de sustentabilidade é obtido calculando-se a área de gráfico tipo radar, sendo esse gerado através da plotagem dos indicadores agregados pelo ângulo entre os raios dos triângulos. Daniel (1999) tomando como base o método proposto por Calório faz algumas adaptações nas fórmulas utilizadas pela autora para simplificar o cálculo da sustentabilidade e facilitar a compreensão geral do sistema de obtenção do Índice de Sustentabilidade. Entretanto, o método proposto neste trabalho para calcular o índice de sustentabilidade (IS), proposto por Daniel (1999), denominado alternativo, quanto ao de Calório (1997), denominado original, parece apresentar problemas quando não utilizado corretamente a escolha de índices.

Palavras-chave: Bacia Hidrográfica; Indicadores de Sustentabilidade; Gráfico de Radar.

\section{Dimensions of sustainability in watershed: an application of the 'radar chart' to Verruga River/BA}

\begin{abstract}
The basin is a place of intrinsic relations of human environment and the natural environment. Therefore, the analysis and evaluation of environmental indicators is essential for the formulation of proposals for sustainable policies for proper management of watersheds. This work was intended to calculate and analyze the sustainability index Mole River basin. For data collection and evaluation of this index on the theme of sustainability, Calório (1997) develops a methodology for calculating the value of sustainability indices is obtained by calculating the area chart type radar, this being generated by plotting the aggregate indicators by the angle between the radii of the triangle. Daniel (1999) building on the method proposed by Calório, makes some adjustments in the formulas used by the author to simplify the calculation of sustainability and facilitate the general understanding of the procurement system Sustainability Index. However, the method proposed in this paper to calculate the sustainability index (SI), proposed by Daniel (1999), called alternate, as to the Calório (1997), original called, seems to present problems if not used correctly choosing indexes.
\end{abstract}

Keywords: Watershed; Sustainability Indicators; Radar Chart.

Topic: Planejamento, Gestão e Políticas Públicas Ambientais

Reviewed anonymously in the process of blind peer.

Rubens Jesus Sampaio (iD

Universidade Estadual do Sudoeste da Bahia, Brasil

http://lattes.cnpq.br/8116836060125065

http://orcid.org/0000-0003-4185-8479

ri.sampaio@uol.com.br

Juliana Oliveira Santos (iD

Universidade Federal da Bahia, Brasil

http://lattes.cnpq.br/3068173121242996

http://orcid.org/0000-0002-1632-6641

uli.oliveira.santos@gmail.com

Dirlêi Andrade Bonfim

Faculdade Independente do Nordeste, Brasil

http://lattes.cnpq.br/8700071457395895

dirleibonfim@gmail.com
Received: $10 / 04 / 2018$

Approved: 24/05/2018
Referencing this:

SAMPAIO, R. J.; SANTOS, J. O.; BONFIM, D. A.. Dimensões da sustentabilidade em bacias hidrográficas: Uma aplicação do 'gráfico de radar' à bacia do Rio Verruga/BA. Revista Ibero Americana de Ciências Ambientais, v.9, n.4, p.336-350, 2018. DOI:

http://doi.org/10.6008/CBPC2179-6858.2018.004.0027 


\section{INTRODUÇÃO}

Com a demanda do sistema produtivo globalizado atual, a natureza apresenta sinais de esgotamento e, como ainda não conseguimos construir uma nova sociedade que seja socialmente justa; ambientalmente adequada e economicamente viável; a fome e a miséria por toda parte, dão sinais de que a ideia de progresso do sistema econômico em vigor na maioria dos países, não conseguiu os resultados esperados. Sob a perspectiva ambiental, a produção é sempre uma produção acoplada; nunca se geram apenas os valores de uso desejados, mas também - e sempre - os produtos secundários indesejados. Eles são, sobretudo, as emissões sólidas, líquidas e gasosas nas esferas da natureza.

É impossível monetizar as transformações da natureza causadas pelo homem. A natureza não se deixa avaliar em grandezas monetárias, por isso não dispomos de um critério que nos permita medir e comparar com segurança os custos do impedimento e do conserto de danos ambientais nem dos próprios danos ambientais. Mais difíceis ainda são os limites da avaliação monetária da preservação da natureza e dos danos por ela sofridos quando consideramos a evolução. A partir deste cenário fica evidente que o crescimento atrelado apenas aos aspectos de progresso econômico, não caracteriza a noção de desenvolvimento, que deve extrapolar o domínio da economia para integrar-se a outras dimensões como a social, a ambiental e a institucional. Surge a necessidade de buscar meios pelo qual os recursos ambientais sejam preservados e conservados para as futuras gerações. Daí surgiu o conceito de desenvolvimento sustentável, propagando-se por todos os seguimentos da sociedade.

Na Comissão Mundial para o Meio Ambiente e o Desenvolvimento (CMMAD), também conhecida como Comissão de Brundtland, presidida pela norueguesa Gro Haalen Brundtland, no processo preparatório a Conferência das Nações Unidas - também chamada de 'Rio 92' foi desenvolvido um relatório que ficou conhecido como 'Nosso Futuro Comum'. Tal relatório contém informações colhidas pela comissão destacando-se as questões sociais, principalmente no que se refere ao uso da terra, sua ocupação, suprimento de água, abrigo e serviços sociais, educativos e sanitários, além de administração do crescimento urbano. Neste relatório está exposta uma das definições mais difundidas do conceito: "o desenvolvimento sustentável é aquele que atende as necessidades do presente sem comprometer as possibilidades de as gerações futuras atenderem suas próprias necessidades". Esse atendimento deve apoiar-se em critérios de plena igualdade, justiça e cidadania para toda a humanidade.

Ao se definir desenvolvimento sustentável também está se discutindo o que é sustentabilidade. Para alguns autores como Clovis Cavalcanti sustentabilidade "significa a possibilidade de se obterem continuamente condições iguais ou superiores de vida para um grupo de pessoas e seus sucessores em dado ecossistema" (CAVALCANTI, 2003). Para o autor, as discussões atuais sobre o significado do termo 'desenvolvimento sustentável' mostram que se está aceitando a ideia de colocar um limite para o progresso material e para o consumo, antes visto como ilimitado, criticando a ideia de crescimento constante sem preocupação com o futuro (CAVALCANTI, 2003). 
Para o objetivo de aproximar a compreensão do conceito de sustentabilidade, Sachs (1993) a divide em cinco classificações: a sustentabilidade ambiental, a sustentabilidade econômica, a sustentabilidade ecológica, a sustentabilidade social e a sustentabilidade política. Daly et al. (1995 citados por LOPES, 2001) relacionam três aspectos como constitutivos da sustentabilidade: o ecológico, o econômico e o social. 0 primeiro refere-se à manutenção das características do ecossistema essenciais à sobrevivência do mesmo a longo prazo. O segundo, diz respeito à gestão adequada dos recursos naturais de tal forma a possibilitar a manutenção da atividade econômica. $O$ terceiro, considera a distribuição adequada dos custos e benefícios entre os indivíduos da população atual (equidade intrageracional) e entre esta geração e as futuras (equidade intergeracional).

Shumarcher (CMMAD, 1991), que classifica somente em sustentabilidade ambiental, econômica e pessoal e refere-se ao uso racional dos recursos. Neste trabalho adota-se a definição de Sachs - presente no texto da Agenda 21 Brasileira - que define sustentabilidade social e política separadamente, fazendo também referência ao uso racional dos recursos no enfoque da sustentabilidade econômica.

O modelo de desenvolvimento adotado pelos países atualmente está voltado apenas para o setor produtivo que privilegia a área econômica. No Brasil, há um processo de urbanização equivocado, concentrando população e industrias nas regiões metropolitanas dos grandes centros urbanos. Como isto, as bacias hidrográficas localizadas nestes centros urbanos são fortemente antropizadas, sofrendo severas pressões sobre o meio ambiente com consequente alteração da qualidade ambiental da área da bacia devido à falta de gerenciamento de uso e ocupação ordenada do solo, ocasionando poluição das águas, do solo e do ar, a disposição inadequada dos resíduos sólidos, lançamento de efluentes líquidos residenciais e industriais nos corpos hídricos, sem o devido tratamento; a ocupação de áreas de risco e atividades clandestinas de mineração, a supressão da cobertura vegetal, erosão e desertificação do solo, trazendo consequências à população local.

Dentro desse cenário, na tentativa do equacionamento de questões ambientais relacionadas à água no território nacional, foi criada no Brasil a Lei 9.433 de 1997 criando o Sistema Nacional de Gerenciamento de Recursos Hídricos, pelo ministério do meio ambiente, para programar a gestão compartilhada do uso da água e tem como fundamento a bacia hidrográfica como unidade territorial para implantação da Política Nacional de Recursos Hídricos. Sob a ótica da gestão ambiental mais integrada, essa Lei procura integrar instrumentos, que darão suporte ao planejamento ambiental, territorial e ao gerenciamento dos recursos hídricos brasileiros. Por este ângulo, observa-se uma forte correlação entre desenvolvimento sustentável e a gerenciamento integrado dos recursos hídricos, resultando em desenvolvimento econômico local aliado à proteção aos recursos naturais. Neste contexto e dada a complexidade da temática ambiental, para que se obtenha um desenvolvimento sustentável local atrelado à gestão dos recursos hídricos, se torna essencial a formulação de indicadores ambientais para a avaliação e medidas que nortearão as tomadas de decisões no gerenciamento de políticas públicas em direção à sustentabilidade. Pois,

A dinâmica da vida e seus relacionamentos ocorrem simultaneamente, e os indicadores têm como função apresentar o que está acontecendo nas esferas social, ambiental e econômica, podendo antecipar acontecimentos, de forma que podem prover uma advertência, a tempo 
de prevenir danos econômicos, sociais ou ambientais. Eles também são ferramentas importantes para comunicar ideias, pensamentos e valores. (GUIMARÃES, 2008)

O Internet Engineering Task Force (IETF) entende que: "um indicador fornece uma pista para uma matéria de largo significado ou torna perceptível uma tendência ou fenômeno que não é imediatamente detectável. Um indicador é um sinal ou sintoma que torna algo conhecível com um razoável grau de certeza". O desenvolvimento só poderá ser sustentado na capacidade de suporte do meio. Moraes et al. (2000), entendem que "o sistema ambiental é semelhante a um sistema vivo que necessita do ar, energia e matéria e ao mesmo tempo 'excreta matérias e tecnologias'. Complementam os mesmos autores:

O balanço desses inputs e outputs, entre o sistema natural e o antrópico, é que irá gerar os indicadores da qualidade do sistema ambiental como um todo, devendo-se levar sempre em consideração o 'equilíbrio' dinâmico do ambiente com processos que se modificam a cada instante. Para a compreensão desse metabolismo complexo torna-se necessária a integração de dados, de conhecimentos, de técnicas, de pesquisadores, de setores e de órgãos. (MORAES et al., 2000)

Atualmente os indicadores já são considerados ferramentas úteis para diagnosticar e monitorar a qualidade ambiental de uma determinada área, acompanhando os sinais por eles evidenciados, norteando o gerenciamento dos recursos dessa área evitando-se a degradação dos recursos naturais.

A importância de um sistema de indicadores de sustentabilidade está ligada à simplicidade que essa ferramenta apresenta ao lidar com as interações que ocorrem no meio ambiente, traduzindo um sistema complexo que envolve o meio físico, a natureza e as relações sociais, em unidades de informação que expressam de forma qualitativa ou quantitativa esses fenômenos. (GUIMARÃES, 2008)

Entende-se, contudo, que a sustentabilidade de um sistema depende da sua conceituação e dos parâmetros que o compõe, ambos variáveis no tempo e no espaço. Nesse sentido, a mensuração de níveis de sustentabilidade constitui-se como uma atividade indispensável para o planejamento, acompanhamento e avaliação de projetos (privados e públicos) e ações voltadas para a promoção do desenvolvimento sustentável.

Desta forma, Moura et al. (2004) define-se indicador de sustentabilidade como: "um conjunto de parâmetros que permita medir as modificações antrópicas num determinado sistema e comunicar, de forma simplificada, o estado deste sistema em relação aos critérios e as metas estabelecidas para avaliar a sua sustentabilidade". Um indicador pode conter um ou vários parâmetros que podem ser considerados isoladamente ou combinados entre si, mas o conjunto de indicadores deve expressar as inter-relações entre eles que possam afetar a sustentabilidade do sistema. Normalmente, o indicador fornece um índice que "corresponde a um nível b superior de agregação onde, depois de aplicado um método de agregação aos indicadores, é obtido um valor final".

Sendo a bacia hidrográfica de um determinado rio ou curso de água, designada pela região cujas águas das chuvas escorrem para esse mesmo curso de água e seus afluentes e subafluentes que, segundo Martins et al. (2003), dá-se através dos desníveis dos terrenos que orientam os cursos da água, sempre das áreas mais altas para as mais baixas. Representa um local de relações intrínsecas do meio antrópico e o meio natural, onde o primeiro meio citado seja composto de subsistemas que podem ser identificados como social econômico e político, o meio natural apresenta como subsistema o ambiental, que engloba a fauna, a flora 
e os recursos naturais. Para avaliar essas interações, a proposta desse trabalho é aplicar a metodologia do 'gráfico de radar', um método de avaliação da sustentabilidade, desenvolvido e testado por Calório (1997, citado por DANIEL, 1999) e simplificado por Daniel (1999), à bacia hidrográfica do Rio Verruga no Estado da Bahia.

\section{REVISÃO TEÓRICA}

\section{A área de estudo}

A bacia do Rio Verruga tem como rio principal, o rio de igual nome de sua bacia: o Rio Verruga é um afluente do Rio Pardo, nascendo na Serra do Periperi, em Vitória da Conquista e desaguando no Rio Pardo, próximo a cidade de Itambé. Possuindo $57,5 \mathrm{~km}$ de comprimento, situando entre os paralelos 140 49' e 15o $16^{\prime}$ de latitude sul e os meridianos 40 $32^{\prime}$ e $40 \circ 55^{\prime}$ de longitude W. Os afluentes mais importantes são: o riacho Santa Rita, Córrego Lagoa de Baixo e Rio Periquito, Córregos Leão, Jeribá, Córrego do Moreira e rios D’Água Fria, dos Canudos, Santa Maria, riacho José Jacinto, córrego Riacho Seco e riacho da Areia. O seu curso d'água tem como limites os municípios de Vitória da Conquista, Barra do Choça e parte do município de Itambé, ocasionando uma bacia de 918 km² de área (ROCHA, 2008). A Bacia Hidrográfica do Rio Verruga, localizada no sudoeste do estado, é uma sub-bacia do Rio Pardo (Figura 1) que faz parte da Região Hidrográfica Nacional do Atlântico Leste. Caracteriza-se por uma área de rochas cristalinas antigas que, no seu alto curso encontram-se as rochas detríticas tercio quaternárias e, no baixo curso, as rochas carbonáticas proterozóicas. Região caracterizada pela forte incisão da drenagem, com expressivos níveis de dissecação e, por conseguinte, declividades acentuadas, com cotas altimétricas que vão desde 950m no alto Rio Verruga até altitude de 500m no baixo rio Verruga, cujos solos são dos tipos latossólico e textural respectivamente. Segundo Benedictis (2007) o solo da região do alto Rio Verruga caracteriza-se:

Os solos que se desenvolvem na área são do tipo Latossolo Vermelho-Amarelo, sendo solos minerais, não hidromórficos, profundos, com baixa fertilidade natural, com avançado grau de intemperismo e boa porosidade, com sequência de horizontes $A$, B e C e com pouca diferenciação entre eles, variando do vermelho ao amarelo com tons intermediários.

A região caracterizada como baixo Rio Verruga, segundo Rocha (2008), “próximo à Itambé, ocorrem, solos do tipo Argissolo, associado com o Brunizém Avermelhado e solos Aluviais". Região com predominância do clima Úmido a Subúmido, com totais pluviométricos que variam entre 1000 a 1500 mm anuais e vegetação remanescente da Mata Atlântica, Mata de Cipó e Caatinga. A região do Planalto de Vitória da Conquista que cultiva café é a região de maior índice pluviométrico, cultivando café de sequeiro, desde a faixa de $1.100 \mathrm{~mm}$ até a faixa de $1500 \mathrm{~mm}$, nas outras áreas o café é cultivado apenas irrigado, sendo essa prática muito pouco desenvolvida, devido poucos recursos hídricos da região.

A região do Planalto de Vitória da Conquista encontram-se plantações de "café, milho, cana de açúcar, banana, feijão, mandioca" (LAURO, 2011), eucalipto e pastagens para criação de gado, além das atividades de mineração, destacando-se as "jazidas de minérios que ainda não são tecnicamente aproveitáveis, mas já se constata a existência de 387 jazidas minerais, 21 minas em exploração e 18 garimpos em atividade. Minerais como a diamita, mármore, cianita, quartzo, granito, manganês, bentonita" (JESUS, 
2010). No município de Itambé além da agricultura de subsistência, há uma extensiva criação de gado e atividades de mineração. Nesta região extrai-se berilo, calcário, cristal de rocha, feldspato, fluorita, nióbio, columbita, caulim, pegmatitos, cristais transparentes, cristal róseo, águas marinhas, ametista, micas, amianto e barita.

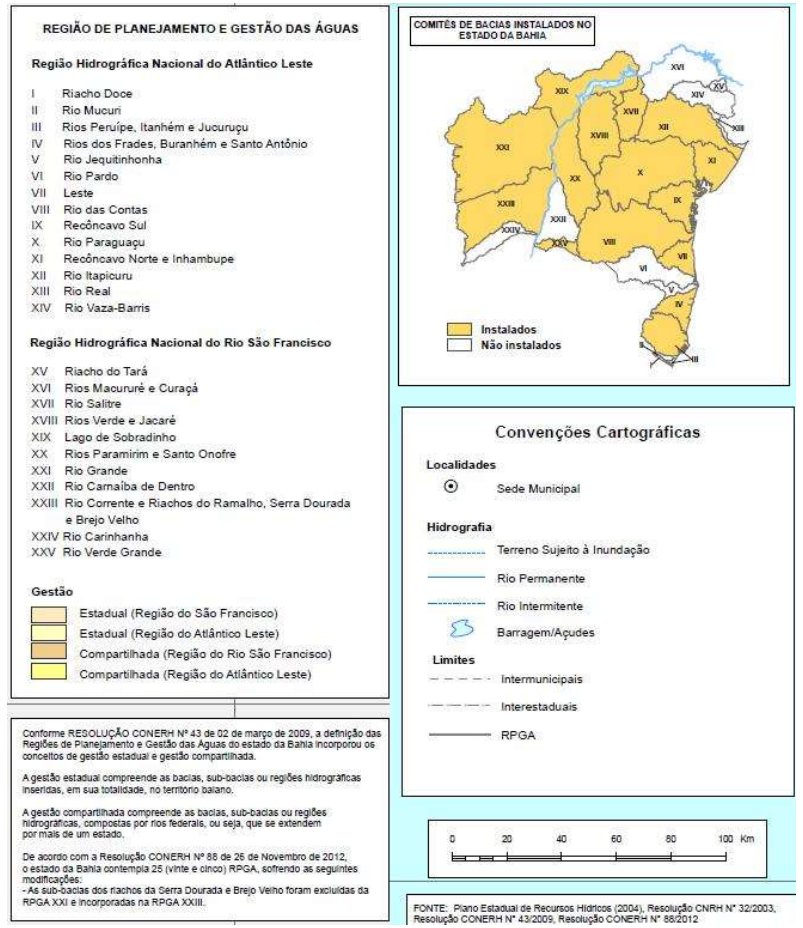

Figura 1: Divisão Hidrográfica do Estado da Bahia. Fonte: Bahia/INEMA (2013).

Trata-se de uma bacia acentuadamente antropizada, onde os principais municípios são Vitória da Conquista e Itambé com densidades demográficas de 90,11 hab/ $/ \mathrm{Km}^{2}$ e 16,02 hab/ $/ \mathrm{Km}^{2}$, cujo somatório é $106,13 \mathrm{hab} / \mathrm{Km}^{2}$ o que representa valor bastante superior à média do estado que é da ordem de 24,82 hab/Km² (IBGE, 2010). Estes dois municípios possuem o índice de Firjan, no ano de 2010, de 0,6867 para Vitória da Conquista e de 0,5028 para o município de Itambé. Ficando a cidade de Vitória da Conquista com índice de Firjan superior à média do Estado da Bahia neste referido ano, que foi de 0.6803 . O índice de Firjan de desenvolvimento municipal acompanha os municípios brasileiros em três áreas: emprego e renda, educação e saúde. Varia de 0 a 1 , quanto mais próximo de 1 , maior o desenvolvimento da localidade (SISTEMA FIRJAN, 2010).

\section{METODOLOGIA}

Este trabalho teve a intenção de calcular e analisar o índice de sustentabilidade da bacia hidrográfica do Rio Verruga. Para obtenção dos dados e avaliação deste índice relativo à temática da sustentabilidade, Calório (1997) desenvolve uma metodologia de cálculo em que o valor dos índices de sustentabilidade é obtido calculando-se a área de gráfico tipo radar, sendo esse gerado através da plotagem dos indicadores agregados pelo ângulo entre os raios dos triângulos. Daniel (1999) embasa-se no método proposto por Calório, faz algumas adaptações nas fórmulas utilizadas pela autora para simplificar o cálculo da sustentabilidade e facilitar a compreensão geral do sistema de obtenção do Índice de Sustentabilidade. 
Utilizamos desta simplificação, proposta por Daniel (1999), para analisar a sustentabilidade de uma determinada realidade de maneira pontual. A seleção dos indicadores para composição dos cálculos foi padronizada pela disciplina Instrumentos de Planejamento e Gestão Ambiental do Programa de Doutorado em Desenvolvimento e Meio Ambiente (PRODEMA) em maio de 2012 na UFS. Além da padronização dos indicadores a coleta dos dados ficou restrita ao site do Instituto Brasileiro de Geografia e Estatística (IBGE).

Conforme esta metodologia, os dados serão trabalhados em ajuste com um conjunto de indicadores ambientais, econômicos e sociais adaptados para este trabalho. Aferindo-se três indicadores para cada classe (ambiental, social e econômica), conforme Quadro 1. A quantificação dos indicadores permitirá a plotagem dos resultados em gráfico do tipo radar. Para Guijt (1999), o indicador constitui-se numa característica quantitativa ou qualitativa de um processo ou atividade acerca dos quais se deseja mensurar as alterações ocorridas, de tal forma que se configura num instrumento para comunicar processos, fatos ou tendências complexas a um público mais amplo.

Quadro 1: Indicadores de Sustentabilidade para a Bacia do Rio Verruga.

\begin{tabular}{|c|c|c|c|}
\hline DIMENSÕES & PADRONIZAÇÃO & INDICADORES & $\begin{array}{l}\text { FONTE E ANO DOS DADOS UTILIZADOS } \\
\text { PARA A CONSTRUÇÃO DOS IDS }\end{array}$ \\
\hline \multirow{3}{*}{ Ambiental } & A1 & Taxa de domicílios com acesso a água tratada & IBGE (2010) \\
\hline & A2 & $\begin{array}{l}\text { Taxa de domicílios com acesso ao esgotamento } \\
\text { sanitário }\end{array}$ & IBGE (2010) \\
\hline & A3 & Taxa de domicílios com coleta de lixo & IBGE (2010) \\
\hline \multirow{3}{*}{ Social } & S1 & Taxa de Mortalidade Infantil & SESAB (2010) \\
\hline & S2 & $\begin{array}{l}\text { Taxa de analfabetismo da população de } 15 \text { anos } \\
\text { ou mais de idade }\end{array}$ & IBGE (2010) \\
\hline & S3 & Taxa de Crescimento da População (2000 a 2010) & $\begin{array}{l}\text { IBGE (2000) } \\
\text { IBGE (2010) }\end{array}$ \\
\hline \multirow{3}{*}{ Econômica } & E1 & PIB per capita a preços correntes (2010) & IBGE (2010) \\
\hline & E2 & $\begin{array}{l}\text { Taxa da população economicamente ativa de } \\
\text { idade igual e/ou superior a } 10 \text { anos }\end{array}$ & IBGE (2010) \\
\hline & E3 & Taxa de domicílios com energia elétrica & IBGE (2010) \\
\hline
\end{tabular}

Tratando-se de uma bacia hidrográfica, para a verificação da sustentabilidade local, optou-se por analisar as maiores cidades localizadas no alto, médio e baixo curso desta bacia: Vitória da Conquista é a maior cidade localizada no alto curso e a cidade de Itambé a segunda maior, estando localizada no baixo curso da bacia citada. Foram analisados três indicadores para cada dimensão (ambiental, social e econômica), totalizando em nove indicadores ao todo para as cidades de Vitória da Conquista e Itambé. Em se tratando de municípios cujas áreas territoriais não se encontravam totalmente inseridas na bacia estudada, optou-se, para a obtenção do valor de cada indicador analisado, pela média ponderada dos valores dos indicadores encontrados nas cidades citadas.

Nesta metodologia cada um dos eixos do gráfico corresponde a um dos indicadores e o somatório das áreas formadas entre os indicadores adjacentes corresponde ao índice de sustentabilidade. A análise gráfica é feita de forma comparativa: quanto maior o índice de sustentabilidade (IS), maior a sustentabilidade das atividades analisadas.

De posse dos valores dos indicadores selecionados para avaliar a sustentabilidade local, os autores, Daniel et al. (2001), recomendam a transformação dos valores dos indicadores para um valor padronizado, 
que permite eliminar os efeitos de escala e de unidade de medida, obtendo-se um gráfico útil em monitoramentos ao longo prazo. Em cada dimensão foram avaliados cinco critérios de sustentabilidade: produtividade, equidade, estabilidade, autonomia e resiliência. Todos os parâmetros receberam o mesmo peso na determinação do índice de sustentabilidade:

$$
\mathrm{Vpn}=\frac{5+(\mathrm{xn}-\overline{\mathrm{x}})}{\mathrm{s}}
$$

Onde: Vpn = valor do indicador padronizado; $\mathrm{xn}=$ valor original do indicador $\mathrm{n}$;

$\overline{\mathrm{X}}=$ valor médio de todos os indicadores;

$\mathrm{S}=$ desvio padrão para todos os indicadores; 5 = constante acrescentada por Calório (1997). Obtenção do ângulo formado entre dois indicadores adjacentes:

$$
\propto=\frac{360}{\mathrm{~N}} \times \frac{\pi}{180}
$$

Onde: $\propto$ = ângulo formado entre dois indicadores, em radianos; $\mathrm{N}=$ número total de indicadores; $\frac{\pi}{180}=$ fator de transformação de graus em radianos.

Cálculo da área de cada triângulo do gráfico de radar, a partir do valor padronizado de dois indicadores adjacentes e do ângulo $\propto$.

Obtenção do ângulo $\beta$ :

$$
\beta=180-90-\alpha
$$

Cálculo da área do triângulo.

$$
S n=\frac{(|V p n| x \cos \beta \times|V p n+1|)}{2}
$$

Cálculo do índice de sustentabilidade.

$$
\text { IS }=\sum_{n=1}^{N} S n
$$

\section{RESULTADOS E DISCUSSÃO}

\section{Dimensão ambiental}

\section{A1-Taxa de domicílios com acesso a água tratada}

Através de dados do IBGE (2010), obtiveram-se os dados da tabela 1. 
Tabela 1: Taxa de domicílios com acesso à água tratada.

\begin{tabular}{|l|l|l|l|}
\hline Cidade & $\begin{array}{l}\text { Domicílios com acesso a água } \\
\text { tratada }\end{array}$ & $\begin{array}{l}\text { Total de } \\
\text { domicílios }\end{array}$ & $\begin{array}{l}\text { Percentual de domicílios com acesso à água } \\
\text { potável }\end{array}$ \\
\hline $\begin{array}{l}\text { Vitória } \\
\text { Conquista }\end{array}$ & 75488 & 86460 & $82 \%$ \\
\hline Itambé & 4977 & 6092 & $87 \%$ \\
\hline
\end{tabular}

Fonte: IBGE (2010).

Considerando que $70 \%$ do município de Vitória da Conquista e 30\% do município de Itambé estão inseridos na bacia do Rio Verruga, para o cálculo deste índice de sustentabilidade utilizou-se de média ponderada, onde o $70 \%$ é representado pelo peso 7 e $30 \%$ pelo peso 3 :

Média ponderada $=0,82 \times 7+0,87 \times 3 / 10=0,85407$ ou $85,4 \%$

\section{A2-Taxa de domicílios com acesso ao esgotamento sanitário}

Tabela 2: Domicílios com acesso ao esgotamento sanitário.

\begin{tabular}{|l|l|l|l|}
\hline Cidade & Domicílios com acesso ao esgotamento sanitário & Total de domicílios & Percentual de esgotamento \\
\hline Vitória da Conquista & 42800 & 86460 & $49,5 \%$ \\
\hline Itambé & 0 & 6092 & $0 \%$ \\
\hline
\end{tabular}

Fonte: IBGE (2010).

Média ponderada $=0,495 \times 7+0 \times 3 / 10=0,3465$ ou $34,65 \%$

\section{A3- Taxa de domicílios com coleta de lixo}

Tabela 3: Coleta de Lixo.

\begin{tabular}{|l|l|l|l|}
\hline Cidade & Domicílios com acesso à coleta de lixo & Total de domicílios & Percentual de coleta de lixo \\
\hline Vitória da Conquista & 77051 & 86460 & $89 \%$ \\
\hline Itambé & 5094 & 6092 & $83.6 \%$ \\
\hline
\end{tabular}

Fonte: IBGE (2010).

Considerando que $70 \%$ do município de Vitória da Conquista e 30\% do município de Itambé estão inseridos na bacia do Rio Verruga, para o cálculo deste índice de sustentabilidade utilizou-se de média ponderada, onde o $70 \%$ é representado pelo peso 7 e $30 \%$ pelo peso 3 :

Média ponderada $=0,89 \times 7+0,836 \times 3 / 10=0,8738$ ou $87,38 \%$

\section{Dimensão social}

\section{S1- Taxa de Mortalidade Infantil}

Tabela 4: Mortalidade Infantil.

\begin{tabular}{|l|l|}
\hline Cidade & No de óbitos de residentes /1000 nascidos vivos no mesmo local e período- ano 2010 \\
\hline Vitória da Conquista & $22,0 \%$ \\
\hline Itambé & $40,1 \%$ \\
\hline
\end{tabular}

Média ponderada $=0,22 \times 7+0,40 \times 3 / 10=0,274$ ou $27,4 \%$

\section{S2- Taxa de analfabetismo da população de 15 anos ou mais de idade}

Tabela 5: Analfabetos de idade igual e/ou superior a 15 anos.

\begin{tabular}{|l|l|l|l|}
\hline Cidade & Total de analfabetos & Total de domicílios & Percentual de analfabetos \\
\hline Vitória da Conquista & 19761 & 86460 & $6,4 \%$ \\
\hline Itambé & 2211 & 6092 & $9,5 \%$ \\
\hline
\end{tabular}

Fonte: IBGE (2010). 
Média ponderada $=0,064 \times 7+0,095 \times 3 / 10=0,07351$ ou $7,35 \%$

\section{S3- Taxa de Crescimento da População (2000 a 2010)}

Tabela 6: Taxa de crescimento populacional.

\begin{tabular}{|l|l|l|l|}
\hline Cidade & $\begin{array}{l}\text { Ano 2000 } \\
\text { (habitantes) }\end{array}$ & $\begin{array}{l}\text { Ano 2010 } \\
\text { (habitantes) }\end{array}$ & Taxa de crescimento populacional \\
\hline Vitória da Conquista & 262.494 & 306.866 & 1,69 \\
\hline Itambé & 22.175 & 23.089 & 0,41 \\
\hline
\end{tabular}

Fonte: Adaptado de IBGE $(2000,2010)$.

A taxa de crescimento médio anual da população foi calculada pela fórmula:

$$
\mathrm{Tx}=(\mathrm{Pf}-\mathrm{Pi}) / \mathrm{Pi} / \mathrm{T} \times 100
$$

Onde:

Pf - população final; Pi - população inicial; T- período.

Considerando que $70 \%$ do município de Vitória da Conquista e 30\% do município de Itambé estão inseridos na bacia do Rio Verruga, para o cálculo deste índice de sustentabilidade utilizou-se de média ponderada, onde o $70 \%$ é representado pelo peso 7 e $30 \%$ pelo peso 3 :

Média ponderada $=1,69 \times 7+0,41 \times 3 / 10=1,306$ ou $130,6 \%$

\section{Dimensão econômica}

\section{E1- PIB per capita a preços correntes}

Tabela 7: Produto Interno Bruto - PIB.

\begin{tabular}{|l|l|}
\hline CIDADE & PIB a preços correntes (R\$) \\
\hline Vitória da Conquista & 3.469 .179 \\
\hline Itambé & 117.176 \\
\hline
\end{tabular}

Fonte: Adaptado de IBGE (2010).

Considerando que $70 \%$ do município de Vitória da Conquista e $30 \%$ do município de Itambé estão inseridos na bacia do Rio Verruga, para o cálculo deste índice de sustentabilidade utilizou-se de média ponderada, onde o $70 \%$ é representado pelo peso 7 e $30 \%$ pelo peso 3 :

$$
\text { Média ponderada }=3.469 .179 \times 7+117.176 \times 3 / 10=2.463 .578,1
$$

\section{E2- População Economicamente Ativa com Pessoas de 10 anos ou mais de idade}

Tabela 8 - Taxa da população economicamente ativa

\begin{tabular}{|l|l|l|l|}
\hline CIDADE & População economicamente ativa & População total & Taxa da população economicamente ativa \\
\hline Vitória da Conquista & 137901 & 306866 & $44,9 \%$ \\
\hline Itambé & 9104 & 23089 & $39,4 \%$ \\
\hline
\end{tabular}

Fonte: Adaptado de IBGE (2010).

Considerando que $70 \%$ do município de Vitória da Conquista e 30\% do município de Itambé estão inseridos na bacia do Rio Verruga, para o cálculo deste índice de sustentabilidade utilizou-se de média ponderada, onde o $70 \%$ é representado pelo peso 7 e $30 \%$ pelo peso 3 : 
Média ponderada $=0,449 \times 7+0,394 \times 3 / 10=0,4325$ ou $43,25 \%$

\section{E3- Taxa de domicílios com acesso à energia elétrica}

Tabela 9: Taxa de domicílios com acesso à energia elétrica.

\begin{tabular}{|l|l|l|l|}
\hline CIDADE & Domicílios & Domicílios com energia elétrica & Taxa acesso à energia \\
\hline Vitória da Conquista & 86460 & 69025 & $79,8 \%$ \\
\hline Itambé & 6092 & 4735 & $77,7 \%$ \\
\hline
\end{tabular}

Fonte: Adaptado de IBGE (2010).

$$
\text { Média ponderada }=0,798 \times 7+0,777 \times 3 / 10=0,79 \text { ou } 79 \%
$$

Tabela 10: Resultado dos cálculos para obtenção da sustentabilidade.

\begin{tabular}{|c|c|c|c|c|c|c|}
\hline $\begin{array}{l}\text { Indicador } \\
\text { (I) }\end{array}$ & $\begin{array}{l}\text { Dimensão } \\
\text { do } \\
\text { indicador }\end{array}$ & $\begin{array}{l}\text { Dimensão } \\
\text { Padronizada } \\
\text { (Vpn) }\end{array}$ & $\begin{array}{l}\text { Ângulo } \\
\text { formado } \\
\text { entre dois } \\
\text { indicadores } \\
\text { adjacentes }(\alpha) \\
\end{array}$ & $\begin{array}{l}\text { Ângulo formado entre a } \\
\text { linha de altura hn e a } \\
\text { linha de comprimento } \\
\text { do indicador I } n+1(\beta)\end{array}$ & $\begin{array}{l}\text { Cálculo da área } \\
\text { do Triângulo } \\
\text { (Sn) }\end{array}$ & $\begin{array}{l}\text { Cálculo do índice } \\
\text { de } \\
\text { sustentabilidade } \\
\left(\sum_{n=1}^{N} S n\right)\end{array}$ \\
\hline A 1 & $\begin{array}{l}0,85407 \text { ou } \\
85,4 \%\end{array}$ & 9,86395E-07 & 40 & 50 & $1,24985 \mathrm{E}-13$ & \\
\hline A 2 & $\begin{array}{ll}0,3465 & \text { ou } \\
34,65 \% & \\
\end{array}$ & $3,30813 \mathrm{E}-07$ & 40 & 50 & $1,3967 \mathrm{E}-13$ & \\
\hline A 3 & $\begin{array}{ll}0,8738 & \text { ou } \\
87,38 \% & \\
\end{array}$ & 1,10229E-06 & 40 & 50 & $1,38307 \mathrm{E}-13$ & \\
\hline S1 & \begin{tabular}{ll|}
0,274 & ou \\
$27,4 \%$ & \\
\end{tabular} & $3,27584 \mathrm{E}-07$ & 40 & 50 & $8,611 \mathrm{E}-15$ & \\
\hline S2 & $\begin{array}{l}0,07351 \text { ou } \\
7,35 \%\end{array}$ & $6,8629 \mathrm{E}-08$ & 40 & 50 & $4,13745 \mathrm{E}-14$ & \\
\hline S3 & $\begin{array}{ll}1,306 & \text { ou } \\
130,6 \% & \\
\end{array}$ & 1,57399E-06 & 40 & 50 & 1,91833E-06 & \\
\hline E1 & $2.463 .578,1$ & 3,181981289 & 40 & 50 & 0,527117827 & \\
\hline E2 & \begin{tabular}{ll|}
0,4325 & ou \\
$43,25 \%$ & \\
\end{tabular} & 5,32304E-07 & 40 & 50 & $2,02672 \mathrm{E}-13$ & \\
\hline E3 & 0,79 ou $79 \%$ & 9,94054E-07 & 40 & 50 & $3,75565 \mathrm{E}-13$ & \\
\hline & & 9,86395E-07 & & & $\sum_{n=1}^{N} s$ & 0,527119745 \\
\hline
\end{tabular}

Depois da obtenção dos valores dos nove indicadores selecionados, estes foram transformados em um indicador padronizado, para que não ocasionasse valores negativos para os indicadores padronizados, foi calculado a média aritmética dos valores dos indicadores e diminuído do menor valor destes (S2), obtendo o valor de 273.731,36 que será um valor aproximado da constante que foi utilizada na fórmula 1 em substituição da constante 5 desta fórmula. Os resultados obtidos foram plotados para gráfico do tipo radar (Figura 2), que representa os índices de sustentabilidades estudados.

O gráfico não ficou 'radar' e sim uma reta, demonstrando assim uma incoerência na apresentação dos dados. Um exemplo disso é o indicador A3 (Taxa de domicílio com coleta de lixo) que em média ponderada para a bacia do rio Verruga é de $83,88 \%$ dos domicílios, no entanto não reflete no gráfico de radar este percentual considerado bastante expressivo quando em comparação com a média nacional. A mesma coisa acontece com o indicador A1(Taxa de domicílios com acesso a água tratada) onde a média ponderada para acesso no domicílio à água potável é de $85,4 \%$.

É válido salientar que na escolha de indicadores para a dimensão social, são inversamente proporcionais, ou seja, quanto menor a taxa melhor o indicador ao passo que na dimensão ambiental, quanto 
maior a taxa melhor o indicador. Sendo os indicadores inversamente proporcionais provocam uma distorção na análise dos dados para gráfico de 'radar'.

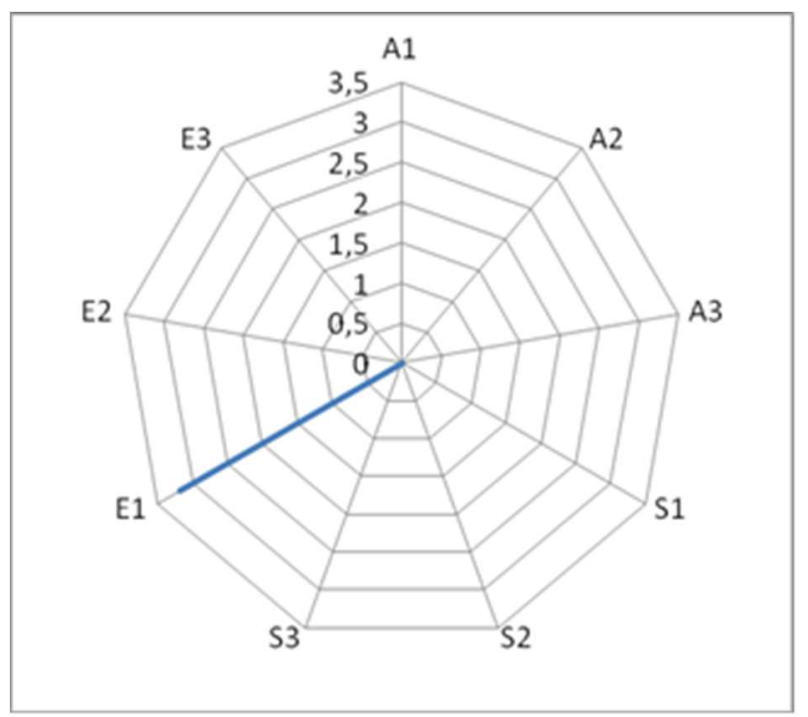

Figura 2: Representação gráfica dos valores calculados a partir dos dados obtidos da pesquisa. Legenda: A1-Taxa de domicílios com acesso à água tratada; A2-Taxa de domicílios com acesso ao esgotamento sanitário; A3- Taxa de domicílios com coleta de lixo; S1- Taxa de Mortalidade Infantil; S2- Taxa de analfabetismo da população de 15 anos ou mais de idade; S3- Taxa de Crescimento da População (2000 a 2010); E1- PIB per capita a preços correntes (2010); E2- População Economicamente Ativa com Pessoas de 10 anos ou mais de idade; E3Consumo de energia per capita.

Segundo Lightfoot et al. (1993), "esse tipo de diagrama produz informações visuais que são úteis para comparar sistemas ao longo do tempo e do espaço" (citado por DANIEL, 1999). Lopes (2001), por sua vez, embora utilize o gráfico tipo radar como instrumento para representar e avaliar visualmente a sustentabilidade de unidades agrícolas calcula os índices de sustentabilidade dessas unidades empregando a média harmônica. O autor justifica sua escolha pontuando que o método de cálculo de índices de sustentabilidade através do cálculo da área de gráfico tipo radar, semelhantemente à média aritmética simples, tende a considerar valores altos, baixos e mesmo nulos, indistintamente, fazendo com que um sistema com valores muito desequilibrados, máximos em uma dimensão e mínimos noutra, por exemplo, alcance índice de sustentabilidade médio, desconsiderando, consequentemente, o equilíbrio entre os indicadores e as dimensões da sustentabilidade consideradas.

Contrariamente, a média harmônica registra maiores índices aos sistemas mais equilibrados (LOPES, 2001). Este aspecto pode indicar que a metodologia de gráfico de radar não pode ser utilizada indistintamente para qualquer indicador. A escolha aleatória, no caso deste estudo padronizado para qualquer bacia em estudo, da composição dos indicadores nas dimensões ambientais, sociais e econômica provoca alterações na combinação de seus valores comprometendo a interpretação dos resultados.

A literatura especializada na temática revela que o processo de definição e seleção de indicadores de sustentabilidade é laborioso e complexo, devendo se atentar a algumas questões importantes. Os indicadores precisam ser os mais específicos possíveis, de modo a se evitar ambiguidades e problemas de validade e confiabilidade, devendo incluir: o objetivo ou a meta a ser alcançado; o aspecto a ser medido; o período abrangido e a área física em questão. Além de específicos, devem ser mensuráveis, atingíveis, 
relevantes e oportunos (GUIJT, 1999). Moura (2002) pontua que os objetivos e metas da avaliação devem considerar "o contexto, a definição e os critérios de sustentabilidade adotados, as expectativas dos atores locais e a viabilidade de obtenção das informações".

Esta afirmação fica comprovada na medida em que os indicadores utilizados apresentam índices bons, todavia a positividade não aprece refletida no gráfico. A utilização de indicadores padronizados sem as devidas especificidades necessárias em cada bacia hidrográfica estudada compromete a aplicação do método.

Vê-se que "é a definição de sustentabilidade que vai determinar o que é importante ser medido, quais serão os componentes dos indicadores e como avaliar e interpretar os resultados" (LOPES, 2001). Os indicadores precisam ser os mais específicos possíveis, de modo a se evitar ambiguidades e problemas de validade e confiabilidade, devendo incluir: o objetivo ou a meta a ser alcançado; o aspecto a ser medido; o período abrangido e a área física em questão. Além de específicos, devem ser mensuráveis, atingíveis, relevantes e oportunos (GUIJT, 1999). Moura (2002) pontua que os objetivos e metas da avaliação devem considerar "o contexto, a definição e os critérios de sustentabilidade adotados, as expectativas dos atores locais e a viabilidade de obtenção das informações".

A existência de valores de indicadores elevados e outros baixos fazem com que os pontos do diagrama referentes a estes praticamente ilegíveis ou invisíveis. Pelo fato de no método proposto ter sido utilizado indicadores com padronização é possível que esta metodologia apresente problemas para cálculos em estudos de avaliação.

\section{CONCLUSÕES}

O método proposto neste trabalho para calcular o índice de sustentabilidade (IS), proposto por Daniel (1999), denominado alternativo, quanto ao de Calório (1997), denominado original, parece apresentar problemas quando não utilizado corretamente a escolha de índices. Ficou evidente o caráter sistêmico da escolha e composição dos dados, indicando as relações sinérgicas e de interdependência entre seus aspectos ambientais, sociais e econômicos. Conhecer a realidade do objeto pesquisado possibilita uma avaliação mais detalhada e evita um resultado distorcido.

Isso pode sugerir que os indicadores selecionados para avaliação do desenvolvimento econômico, social e ambiental ao longo da Bacia do Rio Verruga não foram adequados e não levaram em consideração as especificidades das atividades antrópicas e o potencial de preservação dos recursos naturais locais que influenciam decisivamente na qualidade do meio ambiente e por consequência na vida das populações. Embora sejam evidentes os danos causados à bacia do Rio Verruga em toda sua extensão nos municípios de Vitória da Conquista e Itambé com alguns indicadores apresentando resultados satisfatórios, não há reflexo real na composição do gráfico para avaliação que reflita a realidade da bacia.

O conhecimento da realidade estudada é o que torna possível a validação de um método para a área ambiental. Isso ficou evidenciado quando se apresenta indicadores positivos e a realidade ao longo da bacia não foi refletida aparecendo uma imagem distorcida na formatação da área da sustentabilidade para 
construção do 'radar'. Suspeita-se que estes resultados de efeitos contrários ao que se espera no índice de sustentabilidade (IS), ou seja, o aumento em um dado indicador, do qual se espera influência no aumento do IS, pode na verdade reduzi-lo, e vice-versa. Enquanto isto não é analisado, o método deve ser recomendado apenas para avaliações pontuais.

O IS deve ser capaz de mostrar se o processo de desenvolvimento de um determinado sistema está sendo conduzido para a sustentabilidade ou não, de acordo com metas estabelecidas. Na prática, a utilização dos indicadores e a consequente interpretação dos seus resultados permitem: apoiar decisões e processos de gestão; sintetizar informações de caráter técnico/científico, facilitando a sua transmissão; identificar quais as tendências no longo prazo numa forma sintética, preservando o essencial dos dados originais e utilizando apenas as variáveis que melhor servem para utilização por gestores de políticas públicas, grupos de interesse e para o público em geral.

\section{REFERÊNCIAS}

BAHIA. Instituto de Meio Ambiente e Recursos HídricosBahia. INEMA. 2013.

BENEDICTIS, N. M. S. M.. Política ambiental e desenvolvimento urbano na serra do Periperi em Vitória da Conquista/BA. Dissertação (Mestrado em Ciências Sociais) Universidade Federal do Rio Grande do Norte, 2007.

CALÓRIO, C. M.. Análise da sustentabilidade em estabelecimentos agrícolas familiares no Vale do Guaporé. Dissertação (Mestrado em Agricultura Tropical) Universidade Federal de Mato Grosso, Cuiabá, 1997.

CAVALCANTI, C.. Desenvolvimento e natureza: estudos para uma sociedade sustentável. São Paulo: Cortez, 2003.

CMMAD. Comissão Mundial Sobre Meio Ambiente e Desenvolvimento. Nosso futuro comum. 2 ed. Rio de Janeiro: Fundação Getúlio Vargas, 1991.

DANIEL, O.. Definição de indicadores de sustentabilidade para sistemas agroflorestais. Tese (Doutorado em Ciência Florestal) - Universidade Federal de Viçosa, Viçosa, 1999.

DANIEL, O.; COUTO, L.; SILVA, E.; GARCIA, R.; JUCKSCH, I.; PASSOS, C. A. M.. Alternativa a um método para determinação de um índice de sustentabilidade. R. Árvore, Viçosa, v.25, n.4, p.455-462, 2001.

GUIJT, I.. Monitoramento participativo: conceitos e ferramentas práticas para a agricultura sustentável. Rio de Janeiro: AS-PTA, 1999.

GUIMARÃES, L. T.. Proposta de um sistema de indicadores de desenvolvimento sustentável para bacias hidrográficas. Tese (Doutorado em Ciências em Planejamento Energético) Universidade Federal do Rio de Janeiro, Rio de Janeiro, 2008.

IBGE. Instituto Brasileiro de Geografia e Estatística. Cidades. 2000.

IBGE. Instituto Brasileiro de Geografia e Estatística. Censo Demográfico: 2010. Rio de Janeiro: IBGE, 2010.
JESUS, R. B.. Os recursos naturais e a sua exploração na formação territorial do município de Vitória da Conquista/BA. Enciclopédia Biosfera, Goiânia, v.6, n.9, 2010.

LAURO, A. D.. Análise do uso e ocupação da terra em Vitória da Conquista, Barra do Choça e planalto: estudo das transformações socioambientais a partir da implantação da lavoura cafeeira (1970 a 2008). Dissertação (Mestrado em Geografia) - Universidade Federal da Bahia, 2011.

LOPES, S. B.. Arranjos institucionais e a sustentabilidade de sistemas agroflorestais: uma proposição metodológica. Dissertação (Mestrado em Desenvolvimento Rural) Universidade Federal do Rio Grande do Sul, Porto Alegre, 2001.

MARTINS, R. C.; VALÊNCIO, N. F. L. S.. Uso e gestão dos recursos hídricos no Brasil. Desafios Teóricos e Políticas Institucionais. São Carlos: Rima, 2003.

MORAES, L. A. F.;SOUZA FILHO, E. S.. Indicadores ambientais e desenvolvimento sustentado. Acta Scientiarum, v.22, n.5, p.1405-1412, 2000.

MOURA, L. G. V.. Indicadores para avaliação da sustentabilidade em sistemas de produção da agricultura familiar: o caso dos fumicultores de Agudo - RS. Dissertação (Mestrado em Desenvolvimento Rural) - Universidade Federal do Rio Grande do Sul, Porto Alegre, 2002.

MOURA, L. G. V.; ALMEIDA, J.; MIGUEL, L. A.. Avaliação de sustentabilidade em agroecossistemas: um pouco de pragmatismo. REDES, Universidade de Santa Cruz do Sul (UNISC), v.9, n.2, p.1-13 2004.

OLIVEIRA, E.. Cartografia temática aplicada à elaboração de cenários urbanos: Estudo de caso - Itambé - Bahia. Dissertação (Mestrado em Desenvolvimento Regional e Meio Ambiente) - Universidade Estadual de Santa Cruz, Ilhéus, 2006.

ROCHA, A. A.. Análise socioambiental da bacia do rio verruga e os processos da urbanização de Vitória da 
Conquista/BA. Dissertação (Mestrado em Geografia) Universidade Federal da Paraíba, João Pessoa, 2008.

SACHS, I.. Estratégias de transição para o século XXI

desenvolvimento e meio ambiente. São Paulo: Nobel, 1993.
SISTEMA FIRJAN. Índice Firjan de Desenvolvimento Municipal (IFDM). 2010.

A CBPC - Companhia Brasileira de Produção Científica (CNPJ: 11.221.422/0001-03) detém os direitos materiais desta publicação. Os direitos referem-se à publicação do trabalho em qualquer parte do mundo, incluindo os direitos às renovações, expansões e disseminações da contribuição, bem como outros direitos subsidiários. Todos os trabalhos publicados eletronicamente poderão posteriormente ser publicados em coletâneas impressas sob coordenação da Sustenere Publishing, da Companhia Brasileira de Produção Científica e seus parceiros autorizados. Os (as) autores (as) preservam os direitos autorais, mas não têm permissão para a publicação da contribuição em outro meio, impresso ou digital, em português ou em tradução. 